\title{
Aportes teóricos para el estudio de la cooperación transfronteriza de unidades subestatales de doble periferia*
}

\begin{abstract}
Stella Juste** Nahuel Oddone $e^{* * *}$

\section{Resumen}

El presente artículo propone un acercamiento teórico al estudio de la cooperación transfronteriza de unidades subestatales de doble periferia. Es decir, las acciones de cooperación transfronteriza de unidades subestatales de la periferia de un Estado que, a su vez, se inserta en la periferia del sistema internacional. El estudio de la cooperación transfronteriza resulta un campo de acción internacional clave para las unidades subestatales en esta condición.

El artículo se compone de tres partes. La primera parte aborda, desde el punto de vista teórico, la conceptualización de doble periferia. El análisis se enfoca en las variables que determinan la doble dependencia: el aislamiento geográfico, la cohesión y movilidad social, la calidad de los liderazgos, la inestabilidad institucional, la capacidad de pensar en un modelo de desarrollo propio, y la articulación productiva y las cadenas de valor. El segundo apartado se dedica a la cooperación transfronteriza de las unidades de doble periferia, como herramienta de superación de esa condición y estrategia de desarrollo subestatal. Por último, se presentan algunas reflexiones finales.
\end{abstract}

Palabras claves: cooperación transfronteriza - unidades subestatales - doble periferia - fronteras corredores bioceánicos

\section{Theoretical contributions for the study of cross-border cooperation in double periphery sub-state units}

\begin{abstract}
This article proposes a theoretical approach to the study of cross-border cooperation of double-periphery sub-state units. That is, the cross-border cooperation actions of sub-state units on the periphery of a State that, in turn, is inserted on the periphery of the international system. The study of cross-border cooperation is a key international field of action for sub-state units under this condition.

The article is made up of three parts. The first part addresses, from a theoretical point of view, the conceptualization of double periphery. The analysis focuses on the variables that determine double dependency: geographic isolation, social cohesion and mobility, the quality of leadership, institutional instability, the ability to think of a model of self-development, and productive articulation and value chains. The second section is dedicated to the cross-border cooperation of the double periphery units, as a tool to overcome this condition and a strategy for sub-state development. Finally, some final thoughts are presented.
\end{abstract}

Key words: cross-border cooperation - substate units - double periphery - borders - bioceanic corridors

TRABAJO RECIBIDO: 30/07/2020 TRABAJO ACEPTADO: 29/09/2020

\section{(c) (i) (2)}

Esta obra está bajo una licencia internacional https://creativecommons.org/licenses/by-sa/4.0/

\footnotetext{
* Los autores agradecen los comentarios y observaciones brindadas por el Doctor Mariano Álvarez (REPIT). Las opiniones aquí expresadas son de estricto carácter personal y no representan aquéllas de sus instituciones de pertenencia.

** Doctora en Relaciones Internacionales, Universidad Nacional de Rosario (UNR, Argentina). Becaria postdoctoral del Consejo Nacional de Investigaciones Científicas y Técnicas (CONICET, Argentina), en la Unidad Ejecutora de Ciencias Sociales y Regionales (UE CISOR), CCT Salta-Jujuy. Miembro de la Red de Expertos en Paradiplomacia e Internacionalización Territorial (REPIT) y del Comité de Provincias en el Plano Internacional del Consejo Argentino de Relaciones Internacionales (CARI). Correo electrónico: stellajuste@gmail.com

Doctor en Estudios Internacionales, Universidad del País Vasco/Euskal Erriko Unibertsitatea (UPV/EHU). Jefe de Promoción e Intercambio de Políticas Sociales Regiones del Instituto Social del Mercosur (ISM) (Paraguay), e investigador asociado de United Nations University Institute on Comparative Regional Integration Studies (UNU-CRIS) (Bélgica). Correo electrónico: noddone@ismercosur.com
} 


\section{Introducción}

Múltiples teorías han buscado explicar la estructura y el funcionamiento del sistema internacional. Para ello, en general, se toman en cuenta actores internacionales y procesos. Históricamente, el foco de atención estuvo puesto en el Estado como principal actor del sistema internacional. Con el paso del tiempo, fueron surgiendo otras teorías que analizaban la participación de "otros" actores internacionales, como los gobiernos locales y regionales. Estas teorías que buscan comprender el sistema internacional, también conllevan un sistema de valores, visiones e interpretaciones producto de la posición del actor analizado (y por supuesto del/a investigador/a) en la estructura. En el presente artículo se pone la atención sobre las unidades subestatales consideradas como actores internacionales, haciendo especial hincapié en aquellas ubicadas en una situación de periferia, dentro Estados entendidos como periféricos en el sistema internacional, visto desde el paradigma estructuralista o desarrollista. Es decir, se analizarán desde una perspectiva teórica latinoamericana las unidades subestatales de doble periferia y la cooperación transfronteriza que han intentado implementar como estrategia para mitigar su propia condición de doble dependencia.

En América Latina, la Comisión Económica para América Latina y el Caribe (CEPAL), indagó en los motivos del atraso económico de los países latinoamericanos y las debilidades que impedían la modernización y el consecuente desarrollo. Los referentes de CEPAL, entre ellos Raúl Prebisch, entendieron que la dinámica del sistema internacional era definida por un patrón de desarrollo dominante que establecía condiciones de desarrollo en el centro y de subdesarrollo en la periferia (Sunkel y Paz, 1970, p. 69; Prebisch, 2008, p. 28-29). El paradigma estructuralista o desarrollista se consolidó como visión centro-periférica a partir del estudio de la CEPAL titulado "El desarrollo económico de América Latina y sus principales problemas" de Raúl Prebisch (1949). El estructuralismo se convirtió en la herramienta analítica para interpretar la distribución de los incrementos de productividad que derivaban del cambio técnico y la acumulación del capital y elaborar una concepción del desarrollo de alcance mundial (Beteta y Moreno Brid, 2012, p.79).

Desde esta perspectiva, América Latina se insertó en el sistema internacional desde una situación de subordinación respecto de las economías desarrolladas, escenario que la globalización tendió a profundizar ya que no se alcanzó una transformación sustantiva en las estructuras económico-productivas para asimilar la revolución tecnológica (Cimoli, 2005). Así, la existencia de asimetrías en el sistema internacional y la posibilidad que éstas se reproduzcan en el interior de los Estados, permite reflexionar acerca de las estructuras duales que condicionan y caracterizan el accionar internacional de diferentes actores latinoamericanos, incluyendo las unidades subestatales. La inserción internacional de cada Estado proporciona un primer marco de referencia para su desarrollo e, indirectamente, también para sus unidades subestatales.

La globalización y el proceso de transnacionalización de la economía impulsaron el accionar internacional de unidades subestatales (gobiernos locales y regionales), complementando las acciones del Estado, y aprovechando las oportunidades del escenario internacional. En este contexto, el accionar internacional subestatal (paradiplomacia) comenzó a revestir un tímido interés académico entrada la década del ochenta ${ }^{1}$. Durante la década del noventa, se consolidaron algunos estudios vinculados a la cooperación internacional descentralizada y, en la década siguiente, se avanzó en los análisis de redes de colaboración territorial y también aquellas de carácter transfronterizo. Parte de la literatura especializada considera que ciertos gobiernos locales y regionales lograron modificar relativamente sus posiciones periféricas a través de procesos de internacionalización de sus territorios alcanzando

\footnotetext{
${ }^{1}$ A mediados de los ochenta, la paradiplomacia entra en escena cuando se publica el número de otoño de la revista Publius, dedicada a los Estados federales y las relaciones internacionales, considerado uno de los principales clivajes en los estudios paradiplomáticos. En dicha oportunidad, Ivo Duchacek publicó "The International Dimension of Subnational Self-Government" (Duchacek, 1984) donde se introdujo, por primera vez el término "paradiplomacia". Fue también novedoso que este número incluyese casos europeos y latinoamericanos, aunque analizados todos por autores norteamericanos (Kuznetsov, 2014).
} 
cambios sustantivos en sus estructuras productivas (Cornago, 2006). Dentro de estos enfoques, sobre todo de matriz europea, la cooperación transfronteriza es vista como una herramienta clave para la disminución de asimetrías interterritoriales. Esta situación todavía es difícil de comprobar en las unidades subestatales latinoamericanas.

Teniendo en cuenta la existencia de un sistema que delimita áreas centrales y periféricas a través de relaciones dependencia y, a su vez, el papel creciente de las unidades subestatales en la arena internacional, cabe preguntarse: ¿cómo se insertan las unidades subestatales de doble periferia en un sistema internacional y qué papel ofrece la cooperación transfronteriza para modificar esa situación? Este artículo busca contribuir al análisis de la cuestión en el contexto latinoamericano y se compone de tres partes. En una primera, se estudia desde el punto de vista teórico la existencia de una doble periferia. En una segunda parte, se aborda la cooperación transfronteriza de las unidades subestatales de la doble periferia y la relevancia de la infraestructura. Y, por último, las reflexiones finales.

\section{La doble periferia subestatal}

La relación de dependencia centro-periferia del sistema internacional puede observarse replicada hacia el interior de cada Estado. De manera que un Estado del centro puede contar con unidades subestatales centrales y periféricas, así como un Estado periférico puede contar con unidades subestatales en una posición más central o periférica. Si analizamos un Estado periférico, la duplicación de la relación de dependencia en su interior produce la existencia de unidades subestatales de doble periferia. Es decir, unidades subestatales periféricas de un Estado que, a su vez, es periférico en el sistema internacional. Para verificar la existencia de la doble periferia, es oportuno recordar que el estructuralismo de CEPAL planteó que la instancia económica era insuficiente para explicar la periferia y el consecuente subdesarrollo, siendo necesario analizar el sistema ideológico y político del cual una economía forma parte (Ferrer, 2007, p. 432).

Los estructuralistas hicieron hincapié en las condiciones internas que fueron decisivas para que los Estados del centro generasen un progreso técnico y lo difundieran en su tejido productivo. Este cúmulo de condiciones internas necesarias para el desarrollo, es lo que Ferrer denominó "densidad nacional". En el caso de la periferia, las dificultades en los liderazgos territoriales, la inestabilidad gubernamental y la falta de definición de un modelo consensuado de desarrollo tienden a fortalecer las condiciones de dependencia. El centro ejerce la dominación sobre la periferia en virtud de su capacidad endógena de crecimiento, y tal relación se aplica entre los Estados y hacia el interior de estos. Desde el punto de vista político, esta relación de dominación del centro es comprendida como la obediencia dentro de un grupo (las periferias) de mandatos que son específicos (Melo, 1995, p. 215) y limitan las posibilidades de desarrollo económico-productivo en función de la imposición de reglas (tácitas y no).

Dado que las brechas productivas tienen su correlato territorial (CEPAL, 2010) la apuesta por la convergencia productiva supone cerrar las brechas de productividad con los países más competitivos, pero también reducir la heterogeneidad estructural interna en cada uno de los países y en las fronteras (con los socios regionales). Las asimetrías estructurales se originan en factores tales como el aislamiento geográfico, el acceso a la infraestructura regional, el tamaño económico y la integración en los mercados, la calidad de las instituciones, entre otras (Giordano et al., 2004). A estas asimetrías de índole estructural, pueden también añadirse asimetrías que responden a un carácter coyuntural ligado a la posibilidad de acceso de fuentes de financiamiento, ya sea a través de la captación de inversiones extranjeras directas, tratados bilaterales de inversión o de los mercados de deuda (Oddone y Quiroga, 2015).

Dado que el presente análisis aborda las unidades subestatales de doble periferia, se analizan las variables propuestas por Aldo Ferrer para el análisis de la "densidad (nacional)" a las que se incorporan el aislamiento geográfico y las debilidades en la articulación productiva. 


\section{a) El aislamiento geográfico}

El aislamiento geográfico de las periferias respecto de los principales puntos de distribución, producción y consumo representa una condición característica de la relación de dominación centro-periférica. Una aproximación conceptual general de aislamiento geográfico elaborada por Brigand et. al. (2011, p. 22), lo define como "una situación en la cual un lugar, un territorio, y/o una población está separado de su entorno por un conjunto de factores geográficos, económicos, políticos y/o sociales". Esta definición plantea al aislamiento geográfico como factor complejo de abordar.

Siguiendo a Boisier (1998), las razones por las que las unidades subestatales necesitan desarrollar estrategias alternativas son simples ya que lo que complica su desarrollo no es precisamente su tamaño, o su capacidad productiva sino su soledad (p.6). La asociatividad y la articulación de redes se han convertido en una estrategia clave para la promoción del desarrollo, mejorar el liderazgo y fortalecer la estabilidad institucional de las unidades subestatales de doble periferia, así como para mitigar su aislamiento ${ }^{2}$. En este sentido, por ejemplo, para una provincia del norte argentino representa un desafío casi imposible pensar en responder la demanda de un mercado particular, pero cuando se piensa en la región del norte argentino o, más aun, cuando se piensa en la región comprendida entre el norte argentino y chileno, y el sur boliviano, esa respuesta presenta mejores probabilidades de materialización.

Siguiendo a Ciccolella y Mignaqui (2009), el aislamiento de las unidades periféricas respecto de las centrales debe ser entendido, además, desde una doble dimensión: una dimensión clásica, en la que predominan los elementos físicos como la contigüidad espacial, la aglomeración física de objetos emblemáticos del poder económico (bancos, sedes empresariales, edificios inteligentes); y una dimensión reticular, donde los vínculos predominantes son inmateriales (tecnologías de comunicación e informática) y pertenecen al campo de las múltiples relaciones inter-empresariales (p. 92-93).

La existencia de centros y periferias, implica la delimitación de una zona o área central en la que los poderes económicos y políticos se concentran. Las distancias espaciales de las zonas periféricas respecto de las centrales, pensando en la relación entre las unidades subestatales y el Estado, se visibilizan en la brecha tecnológica existente, sedes empresariales, sedes financieras, servicios avanzados, hotelería internacional, centros políticos, centros culturales, y el hecho que las funciones y actividades de comando de la economía nacional se emplazan en las áreas centro del Estado (Ciccolella y Mignaqui, 2009, p. 92) ${ }^{3}$.

\section{b) El grado de cohesión y movilidad social}

Los países periféricos presentan diversas heterogeneidades, entre ellas, aquellas relacionadas a su composición etnográfica que resultan en múltiples voces hacia el interior de un mismo territorio. Este factor representa un desafío para los Estados en cuanto a su capacidad de respuesta frente a las diferentes demandas y, a la vez, a su habilidad para elaborar políticas públicas que se correspondan con la(s) realidad(es) social(es) del territorio. Como sostiene Ferrer (2011): "la cohesión y el bienestar social están fuertemente asociados al ejercicio de la soberanía

\footnotetext{
${ }^{2}$ Numerosos ejemplos se pueden encontrar en la región. Desde las gestiones de Jujuy y Salta (Argentina) y Tarapacá y Antofagasta (Chile) ante sus respectivos Estados para la habilitación de los Pasos Fronterizos de Jama y Sico y la posterior unificación de los controles fronterizos, o la construcción de un puente entre Monte Caseros (Argentina) y Bella Unión (Uruguay) para conectar el área trinacional que constituyen con Barra do Quareím (Brasil).

${ }^{3}$ Es interesante notar que algunas de las variables sugeridas por los autores se vinculan también con el enfoque de la socióloga Saskia Sassen para caracterizar a las "ciudades globales" a través del papel que estos actores "centrales" en el proceso de globalización tienen en materia de servicios financieros, de innovación, de articulación productiva y en la concentración de instituciones líderes (públicas y privadas) en el gobierno de la economía mundial.
} 
en el despliegue de las políticas públicas y, por lo tanto, a la existencia de una relación simétrica no subordinada con el orden mundial" (p. 11).

Siguiendo la óptica estructuralista, el gobierno central y el de las unidades subestatales registran tensiones sociales en algún momento de su proceso de desarrollo; sin embargo, el elemento clave es cómo se gestionan esas tensiones. La participación social en la decisión sobre el camino del desarrollo eleva sus niveles de cohesión e incrementa las oportunidades de movilidad social (Aronskind, 2016, p. 21). Por lo general, en las regiones periféricas, con un entramado social frágil, y liderazgos con características más autoritarias, las oportunidades de movilidad social suelen ser más limitadas al igual que la cohesión social más fracturada (Juste, 2017, p.178). Al analizar específicamente los Estados periféricos, se observan desequilibrios en la desigual distribución de la riqueza, que se traducen en sociedades con escasas posibilidades de movilidad, siendo las unidades subestatales más afectadas, aquellas alejadas de los centros de producción y distribución principales de los países, que coinciden con las capitales nacionales y las unidades subestatales aledañas (Juste, 2017, p.180).

En las unidades subestatales de la doble periferia los niveles de cohesión social y posibilidad de movilidad son menores en relación a las unidades subestatales centrales que presentan mejores condiciones relativas para el desarrollo. Estas características son mayormente visibles en las áreas de frontera que concentran en sus territorios una complejidad reveladora. Así, las combinaciones posibles generan las singularidades de las zonas de fronteras latinoamericanas entendidas como un espacio de confluencia de, al menos, dos territorios con características de doble periferia ${ }^{4}$. De acuerdo con Oliveira y Max (2009) se puede afirmar que "la proximidad geográfica y el distanciamiento étnico, institucional, político, social, cultural y económico crean un gradiente heterogéneo que diferencia una región fronteriza de otra, donde el grado de conflicto y cooperación puede forzar las relaciones de las sociedades que se encuentran all'’” (p 18).

\section{c) La calidad de los liderazgos}

Los liderazgos de un país se componen de numerosos actores, entre los cuales se encuentra la dirigencia política, social, empresarial, cultural-intelectual, etc. Todas ellas intervienen y contribuyen en la conformación del liderazgo de una unidad política, sea esta un Estado o una unidad subestatal. Para el estructuralismo cepalino, la integración y el comportamiento de estos diferentes liderazgos conllevan a que una sociedad posea, o no, la capacidad para gestionar el progreso técnico necesario para su desarrollo. En este sentido, Ferrer (2011) sostuvo que, en orden a revertir la situación de dependencia de los centros, las periferias debían promover procesos de acumulación de capital y tecnología, administrar recursos y sinergias entre los sectores públicos y privados reteniendo la dirección del proceso de desarrollo (p. 12).

En las periferias, estatales y subestatales, los liderazgos tienden a asociarse a los intereses transnacionales, generando que, a veces, los liderazgos periféricos se reduzcan a mantener el statu quo de la relación dominante centro-periférica existente (Aronskind, 2016, p. 23).

En la relación Estado-unidades subestatales, con frecuencia se observa que la puesta en común de objetivos entre la administración gubernamental, el sector privado y las dirigencias sociales parece difícil de alcanzar. En este panorama y ante la necesidad de captar inversiones de

\footnotetext{
${ }^{4}$ Desde la perspectiva de Santos (1999) los territorios pueden ser comprendidos por los tipos de relaciones que expresan. Una lectura posible, de carácter horizontal, podría estar dada por las relaciones que se establecen en el uso y ocupación de ese territorio. Así, un ejemplo, serían las relaciones producto del uso de servicios que brinda, por ejemplo, el gobierno local de una ciudad de frontera en su entorno, los que exigen el desplazamiento periódico de la población hacia centros de salud, educación, comercio especializado, uso servicios públicos y bancarios, entre otros. Y, por otro lado, desde una perspectiva vertical, se da en las relaciones que se establecen, en diferentes grados y formas, en la inserción de este territorio en el contexto global.
} 
origen extranjero que dinamicen las economías regionales, las unidades subestatales periféricas también contribuyen al sostenimiento de la estructura centro-periférica respecto del Estado y del sistema internacional. En este sentido, los liderazgos subestatales de la periferia no sólo pueden responder a la captación de inversiones extranjeras para la explotación de sus recursos naturales sino, además, a los intereses de las unidades del centro del país desde donde se estructura el modelo económico-productivo estatal/nacional.

\section{d) La inestabilidad institucional}

La estabilidad de los sistemas políticos y las instituciones democráticas se ha estudiado, tradicionalmente, en vinculación al desarrollo económico de una sociedad (Traversa, 2016, p. 162). La estabilidad institucional favorece el desarrollo de la gobernanza de una sociedad, y con eso, diseñar las bases para el crecimiento económico y mejorar la calidad de vida de su población. En este aspecto, la funcionalidad de las instituciones democráticas de un sistema político es tan relevante como lo son las condiciones económicas, puesto que contribuyen a la formación de consensos y la toma de decisión del gobierno del Estado y de la unidad subestatal.

Autores como Vargas Hernández, sostuvieron que las debilidades en la estabilidad de las instituciones democráticas evidencian situaciones de crisis de legitimidad, deficiencias económicas e iniquidades sociales al interior de los Estados latinoamericanos (Vargas Hernández, 2007, s/n). Para el autor, la inestabilidad de las instituciones es una característica de las democracias participativas como las latinoamericanas, por ejemplo, que requieren de la implantación de programas de desarrollo para mejorar la gobernabilidad. El elemento clave se encuentra en la incapacidad de las instituciones democráticas para asumir y procesar democráticamente el conflicto. Esta incapacidad puede, en ocasiones, generar crisis de gobernabilidad en tanto los gobiernos - del Estado central o subestatales- no resultan eficientes para resolver problemas de interacción o de acción colectiva, de institucionalización de reglas y procedimientos (Vargas Hernández, 2007, s/n).

En este sentido, las periferias estatales y subestatales se caracterizan por su incapacidad para procesar el conflicto en momentos de crisis, lo que también dificulta los procesos de transformación política que puedan devolver la estabilidad al sistema político subestatal. Lo anterior se materializa en momentos de tensión política que, por ejemplo, impiden a los gobernantes terminar sus mandatos constitucionales.

\section{e) La incapacidad para diseñar un modelo de desarrollo propio}

Ferrer señaló el efecto negativo del pensamiento céntrico para lograr la densidad nacional que conllevara al desarrollo y permitiera romper el patrón de dominación centro-periférico. Uno de los principales obstáculos ocurre cuando alguna parte del liderazgo político y/o empresarial responde o es portador de un pensamiento céntrico, que se manifiesta en el apoyo declarado a los quiebres institucionales, la adhesión acrítica a los lineamientos de los países centrales hegemónicos, entre otras cuestiones (Ferrer, 2011, p.11-12). Prebisch (2008) oportunamente consideró los intereses de las burguesías periféricas que reflejaban los intereses de los países centrales y no los propios.

Cabe preguntar ¿cuál es el modelo de desarrollo de la periferia? Las economías periféricas se distinguen por ser, esencialmente, exportadoras de commodities con un predominio del sector primario y escasa diversificación productiva. Tales rasgos reafirman la dependencia de los centros, entendiendo que el desarrollo debe propiciar una diversidad de exportaciones, a la vez que se logren exportaciones intra-industriales y con mayor contenido tecnológico que permitan la 
creación de otros productos intermedios o finales a través de encadenamientos productivos de carácter estratégicos 5 .

En este sentido, las unidades subestatales periféricas de países periféricos, presentan economías primarias, escasamente diversificadas y ubicadas, mayormente, en las fronteras estatales. Se trata de espacios en donde el impacto de la transformación tecnológica suele llegar de forma tardía y se ve acompañado de los costos del transporte. Con frecuencia, además, ocurre que las unidades subestatales de doble periferia coinciden con los espacios fronterizos del Estado, elemento que complejiza aún más su proyecto de desarrollo.

\section{f) Debilidades en la articulación productiva}

La articulación productiva de las unidades subestatales de doble periferia debería darse por medio de la creación y el fortalecimiento de vínculos y encadenamientos "hacia atrás" y "hacia delante" en cadenas de valor cuyos eslabones pueden encontrarse incluso en unidades subestatales de un país vecino. Sin embargo, las dificultades de conectividad y la falta de infraestructura son obstáculos para el contacto que estimule la articulación productiva al aproximar espacios económicos, reducir barreras físicas al comercio, reducir los costos de transporte y ampliar la dimensión de los mercados (BID, 2011). A su vez, esto también limita la posibilidad de crear mayores oportunidades de empleo para la población local, consolidando una movilidad social y fortaleciendo la cohesión social del territorio (Oddone, 2016b, p. 22-23).

En líneas generales, una cadena de valor transfronteriza comprende todas las actividades que se requieren para que un producto o servicio transite a través de las diferentes etapas (también conocidas como eslabones) de producción, desde su concepción hasta su entrega a los consumidores y la disposición final después de su uso (Oddone, Padilla y Antunes, 2014, p. 77 78). Cada uno de estos eslabones genera valor (o debería generarlo) y se apropia de una retribución en función de su participación y aporte. Sin embargo, esta retribución depende del esquema de gobernanza de la cadena y de la distribución del poder entre los eslabones, la cual depende de la estructura del mercado, el contenido tecnológico y el esquema de comercialización del servicio o producto.

En este marco, las unidades subestatales de doble periferia presentan un tejido económico que gira, principalmente, en el aprovechamiento y exportación de sus recursos naturales sin lograr procesos de agregación de valor in loco (escalamiento industrial). Una mayor participación de las empresas locales en cadenas de valor nacionales, regionales y globales, posibilitaría aumentar su productividad y escalar su contribución en la actual división internacional del trabajo de la economía mundial (Kosacoff y López, 2008) para así modificar la propia condición de periferia.

En América Latina, en general, las estructuras productivas y exportadoras no están orientadas hacia los sectores más dinámicos, de mayor productividad o de alto contenido tecnológico (Cimoli, 2005; Hausmann y otros, 2005; Kosacoff y López, 2008), lo que no genera modificaciones auténticas en la conformación de una estructura de centro-periferia. En términos subestatales, en las unidades de doble periferia, estas condiciones se agudizan por la simplicidad de las economías regionales y los escasos encadenamientos de los circuitos productivos. El desafío para las unidades subestatales de doble periferia, por lo tanto, es alcanzar una mayor participación en cadenas productivas en segmentos de alto valor agregado a partir de la puesta en

\footnotetext{
5 A modo de ejemplo los proyectos radicados en el "triángulo del litio" en Chile, Bolivia y Argentina, muestran la explotación de las riquezas naturales para la obtención de sales de litio en base a inversiones coreanas, australianas, estadounidenses, alemanas y chinas que son directamente exportados sin agregado de valor. Recientemente, las unidades subestatales periféricas en donde se radican los proyectos (Catamarca, Salta y Jujuy en Argentina, Atacama en Chile y Potosí en Bolivia) han puesto en marcha la articulación entre el gobierno, las empresas y las universidades, para promover la formación de cadenas de valor agregado, pero aún no se visibilizan resultados concretos (Juste, 2019, p. 34-37).
} 
marcha de políticas que fortalezcan las capacidades locales de creación y utilización de conocimientos científicos y tecnológicos.

\section{La cooperación transfronteriza de las unidades subestatales de doble periferia}

Pensar la cooperación transfronteriza en clave teórica y desde la periferia implica la delimitación de un espacio específico que trasciende los actores. La doble periferia, como condicionante para el desarrollo de una unidad subestatal, puede ser relativizada a través de la cooperación transfronteriza y el desarrollo de infraestructura. En las fronteras, las unidades subestatales de doble periferia se vinculan con otras de similares características de la región. Al integrase en espacios transfronterizos a través de la cooperación, las unidades de doble periferia construyen intereses transnacionales que les permiten una mayor capacidad de influencia frente al Estado que integran, reposicionándose en la agenda nacional. Probablemente, el primer interés transnacional común es la construcción de infraestructura pues a través suyo se busca una reconfiguración del espacio común y de las relaciones sociales.

En este sentido, el abordaje de la frontera implica reflexionar sobre un espacio geográfico que no deja de ofrecer paradojas. "Es un marco que limita y separa y que apunta sentidos socializados de reconocimiento" (Pesavento, 2006, p.10). Son múltiples los enfoques teóricos que han estudiado la frontera y sus fenómenos. Normalmente, existen superposiciones conceptuales derivadas de los múltiples significados que se dan al límite y a la frontera. La definición de frontera reviste las complejidades derivadas del entrecruzamiento de las nociones de linealidad, que se identifica jurídicamente con el límite, y de zonalidad, con un sentido socioeconómico. En este aspecto, se entiende la frontera como "un espacio de actuación compartida, escenario de una densa trama de relaciones económicas, sociales y culturales" (Oliveros, 2002, p. 3). Como señalan Oddone y Rodríguez Vázquez, las fronteras son territorios de interacción de los cuales participan agentes a ambos lados del límite y que involucra a los gobiernos no centrales. Mientras el límite es una competencia exclusiva del Estado, en la frontera las competencias son compartidas (Rodríguez Vázquez y Oddone, 2015, p 71-72).

Las acciones de cooperación transfronteriza cobran vital importancia al abordar dinámicas territoriales, enfrentar desequilibrios y asimetrías regionales, al estimular la cohesión social y generar capital humano en las unidades subestatales de doble periferia. La cooperación transfronteriza representa una serie de "formas de innovación específicas en relación al espacio, los lugares y las escalas" (Jessop, 2002, p. 37). Entonces, la cooperación transfronteriza se convierte en "una colaboración entre autoridades subestatales más allá de los límites fronterizos nacionales" (Perkmann, 2003, p. 157) que "permite la participación y actuación conjunta y en forma de red de los actores públicos y privados del territorio a ambos lados de la frontera" (RhiSausi y Oddone, 2009, p.37). Como herramienta, la cooperación transfronteriza ofrece a las unidades subestatales de la doble periferia la alternativa de:

(...) acercar cada sector territorial de los pueblos segmentados geopolíticamente; posibilita a estos pueblos, en parte, paliar los efectos de la división artificial que han sufrido y posibilita la intensificación de lazos de diversos planos entre los distintos sectores territoriales, así como potencias las redes de diversa índole (Fernández Majón, 2005, p. 70).

La cooperación transfronteriza ha crecido ya sea por fenómenos espontáneos surgidos desde los territorios o por fenómenos inducidos producto de las dinámicas de la cooperación internacional y, particularmente, producto de la cooperación internacional descentralizada. El intercambio transfronterizo permite la promoción de una cooperación transnacional a través de la generación de información y cursos para la acción conjunta, a la vez que proporciona una plataforma de negociación y acuerdo para la participación de diferentes actores, la fijación y aplicación de normas, así como la canalización de recursos financieros. 
Para las unidades subestatales de doble periferia, la infraestructura de conexión guarda íntima relación con el objetivo de integrarse en espacios regionales transfronterizos que permitan potenciar sus capacidades para alcanzar un mayor grado de desarrollo socio-económico poniendo fin a una serie de brechas y asimetrías, incluso de carácter histórico producto de la fijación de fronteras ${ }^{6}$. En Sudamérica, las unidades subestatales han apostado a trabajar los corredores bioceánicos a través de infraestructura multimodal (carreteras viales, rutas aéreas, líneas ferroviarias, puertos), favoreciendo los intercambios entre distintos puntos territoriales que van desde el océano Pacífico hasta el Atlántico. El trabajo de las unidades subestatales de la doble periferia ha estado en detectar los puntos territoriales estratégicos y buscar alternativas para nuevas obras de estructura que los conecten, o para mejorar las ya existentes. El resultado es un nuevo escenario de inserción para las unidades subestatales de la doble periferia, en donde la mirada no se dirige hacia las unidades subestatales del centro de los Estados, sino en sentido horizontal hacia sus pares transfronterizos (Juste, 2017, p. 180-81).

La conectividad territorial fortalece un mercado subregional que puede potenciar la productividad de las unidades subestatales de doble periferia a partir mayores flujos de producción y consumo con el consecuente aumento del comercio intrarregional. El desarrollo de infraestructuras que aumente o facilite el contacto entre las regiones estimula la articulación productiva porque aproxima espacios económicos, reduce barreras físicas al comercio, reduce los costos de transporte y amplía la dimensión de los mercados (BID, 2012). La articulación productiva transfronteriza puede ser entendida como un proceso que otorga mayor densidad a los flujos de producción y comercio, derivado de la ampliación del mercado regional que es impulsado por la agregación de valor, las complementariedades productivas y la especialización, cuyo instrumento de acción es la infraestructura y la reducción de los costos del transporte.

El espacio fronterizo juega un papel fundamental en la articulación de los actores económicos que pueden producir escalamiento social. Siguiendo la aproximación de Ciccolella (1997) el desarrollo de las nuevas formas de producción y, especialmente, su creciente tendencia a la fragmentación ha potenciado una nueva interpretación de fronteras móviles y fluctuantes al estilo ratzeliano, poco posibles en el pasado. Desde esta perspectiva puede entenderse la confluencia de fronteras-corredores que potencian el fortalecimiento de las regiones fronterizas (o de contacto) en términos de cooperación y articulación productiva. Por lo general, la preexistencia de ciudades gemelas en ambos lados de la frontera y la dedicación a actividades económicas relativamente similares o complementarias permite la construcción de encadenamientos productivos con su consecuente efecto sobre la transformación territorial del área de confluencia. Sin embargo, el análisis de cadenas regionales de valor enfrenta el desafío de la comparabilidad de los datos entre los países socios que en ciertos casos pueden diferir. Un

\footnotetext{
${ }^{6}$ La formación de los Estados-Nación ha simbolizado uno de los procesos de fragmentación territorial (y por tanto de construcción de fronteras) más importante de la historia tras el fin de los Virreynatos de la era colonial y la introducción de las reformas borbónicas españolas y de las reformas pombálicas portuguesas a mediados del siglo XVIII. Las guerras de Independencia, guerras de liberación -por un lado- y guerras de establecimiento de nuevas fronteras -por el otro-, redundaron también en la desorganización de los modelos productivos locales de las nuevas Repúblicas, en la generación de zonas periféricas rezagadas que no lograrían insertarse en el sistema capitalista internacional y en la generación de fuertes cortes territoriales en donde el "yo" pasaba a ser parte del "otro", es decir, que el dentro pasaba a ser parte del fuera (Rhi-Sausi y Oddone, 2009). Macaulay y Bushnell sostienen que: "En comparación con las viejas naciones de Europa occidental, los países latinoamericanos pueden parecer algo artificiales, ya que no pueden ser diferenciados claramente por criterios de lenguaje, de tradiciones históricas e incluso de religión. Brasil y Haití, como antiguas colonias de Portugal y Francia, quedan lógicamente al margen de estas consideraciones. La razón principal que explica la proliferación de las nuevas naciones atiende al desmembramiento del imperio colonial español, en el que cada uno de sus vástagos iniciaría su andadura nacional con un conjunto de leyes e instituciones similares, heredadas del mismo pasado colonial, con la misma lengua (la que hablaban las minorías dominantes que establecieron las nuevas naciones) y, naturalmente, con la misma religión. A menudo se podían establecer contrastes culturales y socioeconómicos mucho mayores entre diferentes regiones de una misma nación que entre esa misma nación y sus países vecinos" (Maculay y Bushnell, 1989, p. 30).
} 
elemento clave para analizar en el intercambio fronterizo es la diversidad regulatoria y legislativa de las que son objeto los territorios que puede limitar o auspiciar la articulación y complementariedad: en las zonas de frontera "la intensidad suele ser acentuada por la diversidad contrastante en términos de contexto económico general, mercado de trabajo, régimen fiscal, disponibilidad de bienes de consumo, y hasta por la disponibilidad del suelo edificable" (Dell'Agnese, 2003, p. 80).

La creciente fragmentación de la producción global y del comercio internacional ha abierto una oportunidad de participar en diferentes cadenas de valor para los países en desarrollo y sus empresas. Sin embargo, no todos los países ni todas las industrias participan con la misma intensidad en los mismos eslabones de mayor valor agregado. Esto significa que es importante examinar de qué manera participa un territorio específico en una cadena productiva que cruza la frontera y se integra con el vecino y cuál papel tiene en la distribución de beneficios que se derivan de las diferentes actividades de la cadena (Oddone, Padilla y Antunes, 2014). La participación en distintos eslabones tiene implicaciones importantes no sólo en términos de organización industrial y de disposición de la infraestructura, sino también en términos de desarrollo económico y social, debido a que las actividades asociadas con cada eslabón tienen distinta intensidad en el uso de recursos (capital, tecnología, mano de obra o recursos naturales), diferente oportunidad de encadenamientos productivos con otros sectores nacionales $\mathrm{y}$, consecuentemente, variadas oportunidades para el desarrollo de capacidades locales y de escalamiento económico y social.

Las cadenas regionales de valor constituyen un proceso de encadenamiento productivo transfronterizo en el que participan dos o más países con afinidad productiva, cercanía territorial y complementariedad comercial, con el objetivo de alcanzar una mayor articulación a partir de la integración de los diferentes eslabones de uno y otro lado de la(s) frontera(s). De esta manera, el valor "es agregado" por diversos países cercanos con características económicas comunes o complementarias (CENPROMYPE, CEPAL y SIECA, 2018, p. 16). El desarrollo de una infraestructura que aumente o facilite el contacto entre las diferentes regiones permite reducir algunas de las asimetrías estructurales mencionadas en el apartado anterior, ya que estimula la articulación productiva por medio de cadenas de valor que aproximan espacios económicos, reducen barreras físicas al comercio y los costos de transporte, atraen inversiones del sector privado, y amplían la dimensión del mercado.

Los encadenamientos regionales ofrecen una oportunidad para la coordinación operativa de dos de los criterios principales que han impulsado la colaboración de las unidades subestatales de doble periferia. Un criterio geo-económico que ha servido de guía a la integración física en las fronteras; y un criterio geo-político que ha guiado el diálogo transnacional e intersectorial (RhiSausi y Oddone, 2009). La construcción o la ampliación de un puente permiten potenciar la movilidad de bienes y personas en un paso fronterizo, pero la movilidad real dependerá de la existencia de los acuerdos institucionales intersectoriales estructurados que permitirán su funcionamiento a partir de diferentes ejemplos de coordinación. A modo de ejemplo, los esfuerzos por instituir las aduanas integradas en los pasos fronterizos ofrecen una condición fundamental para realizar acuerdos entre los Estados centrales que hagan efectiva la movilidad potenciada por la integración física, pero también se necesita del interés de usufructo por parte del sector privado ${ }^{7}$.

\footnotetext{
7 "La sola administración de un puesto fronterizo integrado -que potencie el desarrollo de cadenas de suministro regional mediante la facilitación del comercio- supone un esfuerzo de coordinación importante entre autoridades nacionales a lo interno de cada gobierno, que va desde los encargados de aduanas, tributación interna, seguridad, migración, control sanitario y fitosanitario, entre otros. Adicionalmente, la coordinación entre los países es relevante en la estandarización de procesos, desde los sencillos como la armonización de horarios hasta los complejos como los sistemas de información migratoria" (CENPROMYPE, CEPAL y SIECA, 2018, p. 53). Al mismo tiempo, las unidades subestatales de doble periferia juegan un papel fundamental en estimular el aprovechamiento de estos beneficios, como puede ser una aduana integrada, por los empresarios locales que realizan inversiones y negocios porque si no estos
} 
En general, en las regiones de frontera conformadas por unidades subestatales de doble periferia, se observan dificultades de diálogo y articulación público-privada en tres niveles, es decir, problemas con el diálogo público-público, público-privado y privado-privado. En materia de diálogo público-público se registran fallas de coordinación, lagunas competenciales y solapamiento de funciones que le quitan coherencia y eficacia a la acción pública y, por tanto, competitividad a las cadenas de valor. En los diálogos público-privados, en muchos casos, ha habido intereses contrapuestos o enlazados a partir de la captura de uno u otro que no han permitido sinergias exitosas. El diálogo privado-privado tampoco ha sido necesariamente exitoso ya que en algunos sectores se observa un muy bajo nivel de asociatividad $y$, cuando se han conformado asociaciones, éstas no han conseguido representar necesariamente los intereses de sus afiliados de manera estratégica y con vocación territorial. En general, el diálogo públicoprivado tiene éxito en aquellos contextos en donde en diálogo público-público funciona de manera ágil y donde hay una identificación clara de intereses por parte del sector público y del privado (Devlin y Moguillansky, 2010).

Algunos ejemplos que vale la pena considerar, en América Latina son, la Zona de Integración del Centro Oeste de Sudamérica (ZICOSUR), impulsada por alianzas públicoprivadas de unidades subestatales del norte argentino y chileno, del sur boliviano, peruano y brasileño, y de la totalidad de los departamentos paraguayos; también la Comisión Regional de Comercio Exterior del Noreste Argentino (CRECENEA) y el Conselho de Desenvolvimento e Integracão Sul (CODESUL/FORUM SUL), un mecanismo de diálogo e integración subestatal de carácter histórico $^{8}$; o la región de ATACALAR, compuesta por las provincias argentinas de Catamarca, Córdoba, La Rioja, Tucumán, Santa Fe y Santiago del Estero con la región chilena de Atacama.

\section{Conclusiones}

La configuración de relaciones de dependencia entre centros y periferias en el sistema internacional también se reproduce, al interior de los propios Estados. Esta situación produce unidades subestatales de doble periferia, condicionadas por una doble dependencia. La doble periferia es una situación política de doble dependencia en que se encuentra una unidad subestatal respecto del centro propulsor internacional y al interior del Estado periférico de pertenencia. Esta condición de doble periferia se ve reflejada en una serie de factores tales como el aislamiento geográfico, la ausencia de liderazgos, la inestabilidad de las instituciones, las limitaciones en la cohesión y movilidad social, la incapacidad para pensar un modelo de desarrollo propio, y la debilidad en una articulación productiva del territorio que incluya cadenas de valor transfronterizas.

A estas características de las unidades subestatales de doble dependencia, hay que sumar el hecho que, generalmente, éstas se ubican en territorios de la frontera estatal, es decir, son unidades fronterizas. Dado este hecho de compartir frontera con otras unidades de condiciones similares, la cooperación transfronteriza emerge como una estrategia e instrumento de vinculación que les permite reposicionar sus intereses en la propia escena nacional, la escena internacional y, generar consensos a escala transnacional. En este sentido, se observa que el reposicionamiento de intereses y la creación de consensos se materializan, en una primera etapa, en la demanda de

beneficios serán principalmente utilizados por capitales extranjeros y otras empresas con poca raigambre en el territorio.

${ }^{8}$ Las provincias argentinas que integran la región del NEA más Santa Fe conformaron CRECENEA el 8 de junio de 1984 con el objetivo de promocionar el comercio internacional de la región. Si bien se llevaron a cabo acciones conjuntas con Uruguay y Paraguay, el hecho más significativo fue el Protocolo $\mathrm{N}^{\circ} 23$ Regional Fronterizo, del 19 de noviembre de 1988, con los vecinos estados brasileños Paraná, Rio Grande do Sul, Mato Grosso do Sul y Santa Catarina que, por su parte, integran el CODESUL, por el cual se constituyó un Grupo de Trabajo Permanente entre ambos organismos que fue receptado por las respectivas Cancillerías de Argentina y Brasil. 
mayor infraestructura para conectar los territorios separados de uno y otro lado de la línea fronteriza y así avanzar en términos asociativos.

El camino de la cooperación y desarrollo de las unidades subestatales de doble periferia requiere de un esfuerzo multiactor que implica lograr una mayor cohesión social, con un empresariado local y transnacional que invierta en el territorio, con instituciones capaces de procesar y resolver los conflictos vinculados a la transformación productiva a través del diálogo y la coordinación multinivel público-pública.

Las regiones transfronterizas conformadas por unidades subestatales de doble periferia demuestran que la cooperación entre sus integrantes contribuye a crear nuevas sinergias para el desarrollo. La experiencia de los últimos años de cooperación transfronteriza en América Latina pone de manifiesto los esfuerzos por articular productivamente los diferentes territorios periféricos y evitar el surgimiento de nuevas asimetrías que se reflejan en una suerte de centroperiferia dentro de los Estados y sus procesos de integración regionales, manifiesta en ejes o corredores principales y otros anexos o alejados de los circuitos económico-productivos y comerciales. La dialéctica entre la movilización de los gobiernos subestatales y las políticas nacionales de cohesión territorial necesitan crear círculos virtuosos entre el capital social y la política pública, fortaleciendo las capacidades de desarrollo específicas que los territorios periféricos de frontera demanden. La infraestructura y sus servicios conexos, así como el diálogo público-privado, privado-privado y público-público, actúan como un vehículo para la cohesión territorial, económica y social, por lo que mantener en el tiempo niveles adecuados de inversión en infraestructura es, por tanto, un objetivo socialmente deseable.

La cooperación transfronteriza busca apoyar que las unidades subestatales de doble periferia fortalezcan su grado de cohesión social, mejoren la calidad de sus liderazgos políticos, consoliden la estabilidad institucional y, sobre todo, tengan la capacidad para pensar un modelo de desarrollo propio que les permita escapar de su condición, a la vez que hacen contribuciones para que los propios Estados a los que pertenecen escapen de su situación periférica dentro del sistema internacional a partir de avances sustantivos en la disminución de la heterogeneidad estructural característica de las economías latinoamericanas.

\section{Referencias Bibliográficas}

Aronskind, R. (2016). Aldo Ferrer y la construcción de la densidad nacional. Voces en el Fénix, Buenos Aires, año 7, 54, mayo

Association of European Border Regions (2008). Cooperation between European Borders Regions. Review and Perspectives, Baden-Baden (Germany): Nomos y AEBR

Banco Interamericano de Desarrollo-Instituto para la Integración de América Latina y el Caribe (2011). IIRSA, 10 años después: sus logros y sus desafíos. Buenos Aires (Argentina): BID-INTAL

Beteta, H. y Moreno-Brid, J. C. (2012). El desarrollo en las ideas de la CEPAL. Revista Economía UNAM, México D.F., 9 (27), pp. 76-90

Boisier, S. (1998). Teorías y metáforas sobre el desarrollo territorial. Revista Austral de Ciencias Sociales de Chile, Santiago (Chile), Vol. marzo-agosto, $\mathrm{N}^{\circ} 2$

Boisier, S. (2001). Desarrollo (local): ¿de qué estamos hablando?. En A. Vázquez Barquero y O. Madoery (comp.). Transformaciones globales, instituciones y políticas de desarrollo local, pp. 48-74. Rosario (Argentina): Editorial Homo Sapiens 
Brigand,L., Peuziat, I., Arenas, F., Núñez, A., Salazar, A., y Escobar, H. (2011). Multiplicidad de miradas y valorización del aislamiento geográfico en la Región de Aysén. En F. Arenas, A. Salazar y A. Núñez (edit.). El aislamiento geográfico: ¿problema u oportunidad? Experiencias, interpretaciones y políticas públicas. Santiago de Chile: Pontificia Universidad Católica de Chile

Centro Regional de promoción de la MIPYME (CENPROMYPE), Comisión Económica para América Latina y el Caribe (CEPAL) y Secretaría de Integración Económica Centroamericana (SIECA) (2018). Articulación productiva y cadenas regionales de valor: una propuesta metodológica para la Región SICA. Ciudad de México (México): CEPAL, SIECA y CENPROMYPE

Ciccolella, P. (1997). Redefinición de fronteras, territorios y mercados en el marco del capitalismo de bloques. En I. R. Castello, M. R. Koch, N. Oliveira, N. Otero Schäffer, y T. Strohaecker (org.). Fronteiras na América Latina. Porto Alegre (Brasil): Universidade Federal de Rio Grande do Sul

Ciccolella, P. y Mignaqui, L. (2009). Globalización y transformaciones de la centralidad histórica en Buenos Aires. Centro-h, Organización Latinoamericana y del Caribe de Centros Históricos - OLACCHI, Quito, núm. 3, abril, pp. 91-101

Cimoli, M. (ed.) (2005). Heterogeneidad estructural, asimetrías tecnológicas y crecimiento en América Latina. Santiago de Chile (Chile): Comisión Económica para América Latina y el Caribe (CEPAL) y Banco Interamericano de Desarrollo (BID)

Comisión Económica para América Latina y el Caribe (CEPAL) (2010). La hora de la Igualdad. Brechas por cerrar, caminos por abrir. Santiago (Chile): CEPAL-Naciones Unidas

Comisión Económica para América Latina y el Caribe (CEPAL) (2012). Cambio estructural para la igualdad: una visión integrada del desarrollo. Santiago (Chile): CEPAL-Naciones Unidas

Cornago, N. (2006). Un acercamiento al concepto de eurorregión: dimensiones funcionales y economía simbólica. En F. Letamendia (ed.). Acción colectiva Iparralde-Hegoalde, Madrid (España): Fundamentos.

De Mattos, C. (2002). Transformación de las ciudades latinoamericanas: ¿Impactos de la globalización?. EURE, Pontificia Universidad Católica de Chile, vol. XXVIII, núm. 85, diciembre

Devlin, R. y Moguillansky, G. (2010). Alianzas público-privadas para una nueva visión estratégica del desarrollo. Santiago de Chile (Chile): Comisión Económica para América Latina y el Caribe (CEPAL)

Domingues, J. M. (2012). Desarrollo, periferia y semiperiferia en la tercera fase de la modernidad global. $1^{\circ}$ edición, Ciudad Autónoma de Buenos Aires (Argentina): Centro Latinoamericano de Ciencias Sociales (CLACSO)

Duchacek, I. (1984). The International Dimension of Subnational Self-Government, Publius, 14(4).

Fantin, M.A. (2008). Población, sociedad y salud en la frontera argentino-paraguaya. Asunción (Paraguay): Asociación de Estudios de Población y Fondo para la Población de las Naciones Unidas

Fernández Manjón, D. (2005). El papel de las regiones en la dinámica fronteriza en Europa. Revista CIDOB d'Afers Internacionals, 69. Barcelona (España): Barcelona Centre for International Affairs

Ferrer, A. (1999). La globalización, la crisis financiera y América Latina. En Varios Autores. Tiempos violentos. Neoliberalismo, globalización y desigualdad en América Latina. 
Ciudad Autónoma de Buenos Aires (Argentina): Centro Latinoamericano de Ciencias Sociales (CLACSO)

Ferrer, A. (2007). Globalización, desarrollo y densidad nacional. En G. Vidal y A. Guillón (comp). Repensar la teoría del desarrollo en un contexto de globalización. Homenaje a Celso Furtado. Ciudad Autónoma de Buenos Aires (Argentina): Centro Latinoamericano de Ciencias Sociales (CLACSO)

Ferrer, A. (2010). Raúl Prebisch y el dilema del desarrollo en el mundo global. Revista CEPAL, Santiago de Chile (Chile), No 101

Ferrer, A. (2011). Los problemas fundacionales y la densidad nacional. Revista de ciencias sociales, 3(19), pp. 7-24. Recuperado de RIDAA-UNQ Nacional de Quilmes http://ridaa.unq.edu.ar/handle/20.500.11807/1510

Giordano, P., Mesquita Moreira, M. y Quevedo, F. (2004). El Tratamiento de las asimetrías en los acuerdos de integración regional. Buenos Aires (Argentina): Banco Interamericano de Desarrollo - Instituto para la Integración de América Latina y el Caribe (BIDINTAL)

Haussmann, R., Rodrik, D. \& Velasco, A. (2005). Growth diagnostics. En N. Serra \& J. Stiglitz (eds.). The Washington Consensus Reconsidered: Towards a New Global Governance, New York (USA): Oxford University Press

Jessop, B. (2002). The Political Economy of Scale. En M. Pekermann \& N. Sum (eds.). Globalization, Regionalization and Cross Border Regions. London (United Kingdom): Palgrave Macmillan, Basingstoke

Juste, S. (2017). La condición de doble periferia en unidades subestatales. Revista INTERAÇÕES, 18(4). Campo Grande (Brasil), Universidade Católica Dom Bosco

Juste, S. (2019). La agenda subestatal de las relaciones argentino-chinas. La gestión transnacional de las provincias argentinas durante la administración de Macri. Revista Intellector. Año XV, Vol. XVI, No 32, Julho/dezembro, Rio de Janeiro (Brasil)

Kosacoff, B. y López, A. (2008). América Latina y las cadenas globales de valor: debilidades y potencialidades. Revista globalización, competitividad y gobernabilidad, vol. 2, $\mathrm{N}^{\circ} 1$, Washington, D.C. (USA): Georgetown University

Kuznetsov, A. (2014), Theory and Practice of Paradiplomacy. Subnational governments in international affairs. New York (USA): Routledge

Macaulay, N. y Bushnell, D. (1989). El nacimiento de los países latinoamericanos. Madrid (España): Ed. Nerea

Melo, A., (1995), Compendio de Ciencia Política. Tomo I, Buenos Aires (Argentina): Ediciones Depalma

Oddone, N. (2013) Cooperación regional en el ámbito de la integración fronteriza. XXIV Reunión de Directores de Cooperación Internacional de América Latina y el Caribe. Cooperación Regional en el Ámbito de la Integración Fronteriza, Caracas, SELA, BID, CAF, Grupo de las 77 y Ministerio de Relaciones Exteriores del Salvador (SP/XXIVRDCIALC/Di ${ }^{\circ}$ 12-13)

Oddone, N. (2016a). La paradiplomacia transfronteriza de los gobiernos locales en el MERCOSUR (2003-2013): una aproximación teórica y práctica. Bilbao (España): Universidad del País Vasco / Euskal Herriko Unibertsitatea

Oddone, N. (2016b). Política comercial y cadenas de valor en Argentina contemporánea: ¿una nueva etapa? Revista Puentes. Volumen 17, Número 2, Abril, pp. 22-26

Oddone, N. y Quiroga, M. (2015). Fronteras de América Latina: Infraestructuras y fondos regionales para garantizar condiciones de igualdad. En N. Oddone, M. Quiroga, H. 
Sartori y A. Williner. Pactos territoriales en la construcción de regiones transfronterizas: por una mayor integración a múltiples niveles. Santiago de Chile (Chile): Instituto Latinoamericano y del Caribe de Planificación Económica y Sociales (ILPES), Comisión Económica para América Latina y el Caribe (CEPAL)

Oddone, N. y Rodríguez Vázquez, H. (eds.) (2014). Municipios y cambio climático: hacia la construcción de una agenda de paradiplomacia ambiental. Granada (España): Unión Iberoamericana de Municipalistas y Secretaría General Iberoamericana

Oddone, N., Quiroga, M., Sartori, H. y Williner, A. (2015). Pactos territoriales en la construcción de regiones transfronterizas: por una mayor integración a múltiples niveles. Santiago de Chile (Chile): Instituto Latinoamericano y del Caribe de Planificación Económica y Sociales (ILPES), Comisión Económica para América Latina y el Caribe (CEPAL)

Oddone, N.; Padilla Pérez, R. y Antunes, B. (2014). Metodología del Proyecto CEPAL-GIZ para el diseño de estrategias de fortalecimiento de cadenas de valor. En R. Padilla Pérez (ed.). Fortalecimiento de las cadenas de valor como instrumento de la política industrial. Metodología y experiencia de la CEPAL en Centroamérica. Santiago de Chile (Chile): CEPAL

Oliveira, T.C.M. y Max, C.Z. (2009). As relações de troca em região de fronteira: uma proposta metodológica sob a ótica convencionalista, Geosul, v. 24, n. 47, págs 7-27, jan./jun.

Oliveros, L. (2002). Integración de las Fronteras Andinas. Elementos de una propuesta para el estudio, clasificación y el diseño de cursos de acción en materia de integración y desarrollo fronterizo en los países de la comunidad andina. Documento Informativo. Comunidad Andina, Secretaría General, Abril, SG/ di. Recuperado de: http://intranet.comunidadandina.org/documentos/DInformativos/SGdi439.pdf

Perkmann, M. (2003). Cross Border Regions in Europe. Significance and drivers of regional cross-border cooperation. En European Urban and Regional Studies, 10 (2), pp. 153171

Pesavento, S. (2006). Fronteiras culturais em um mundo planetário - paradoxos da(s) identidade(s) sul-latino-americana(s). Revista del CESLA, 8. Varsovia (Polonia): Uniwersytet Warszawski

Pollock, D. (2006). Raúl Prebisch: la esencia del líder. En. E. Dosman. (Coord.) Raúl Prebisch: el poder, los principios y la ética del desarrollo. 1. ed. Buenos Aires (Argentina): BIDINTAL

Prebisch, R. (1949). El desarrollo económico de América Latina y algunos de sus principales problemas, Santiago de Chile (Chile): CEPAL

Prebisch, R. (2008). Hacia una teoría en transformación. Revista de la CEPAL, Santiago de Chile, $\mathrm{N}^{\circ} 6$

Quintar, J. y Perrén, J. (2016). El gran desafío argentino: entrevista a Aldo Ferrer. Revista Hindustri@, Buenos Aires, Año 10, $\mathrm{N}^{\circ}$ 18, primer semestre

Rhi-Sausi, J. L. y Oddone, N. (2009). Fronteras y cooperación transfronteriza en América Latina: introducción al Proyecto Fronteras Abiertas. En J.L.Rhi-Sausi y D. Conato (coords.). Cooperación Transfronteriza e Integración en América Latina. Roma (Italia), Centro Studi di PoliticaInternazionale e IstitutoItalo-Latino Americano

Rodriguez Vazquez, H. y Oddone, N. (2015). Fertilidad territorial transfronteriza: el papel innovador de la paradiplomacia como fortalecimiento de la integración desde abajo. En Ma. del Rosio Barajas Escamilla, P. Wong-González y N. Oddone (coord.). Fronteras y procesos de integración regional. Estudios comparados entre América y Europa. Ciudad de México (México): Juan Pablos Editor, El Colegio de la Frontera Norte Centro de Investigación en Alimentación y Desarrollo 
Santos, M. (1999). O Território e o saber local: algumas categorias de análise. Cadernos do IPPUR, Rio de Janeiro (Brasil), N. 2, pp. 15-26, Semestral

Sassen, S. (1991). The Global City: New York, London, Tokyo. Princeton (USA): Princeton University Press

Sunkel, O. y Paz, P. (1970). El subdesarrollo latinoamericano y la teoría del desarrollo. $1^{\circ}$ edición, México D.F.: Siglo XXI Editores

Traversa, F (2016). Desarrollo económico y estabilidad democrática: un problema fundamental para la política comparada latinoamericana. Reviste Debates, Porto Alegre, v. 10, n. 1, enero-abril, pp. 161-192

Vargas Hernández, J. (2007). Crisis de gobernabilidad en el Estado-Nación. IX Coloquio Internacional de Geocrítica "Los problemas del mundo actual, soluciones y alternativas desde la geografía y las ciencias sociales", Universidad de Río Grande do Sul, Porto Alegre 